\title{
A phase II, open-label clinical trial on the combination therapy with medium-chain triglycerides and ghrelin in patients with chronic obstructive pulmonary disease
}

\author{
Keisuke Miki ${ }^{1}$ (1) - Seigo Kitada ${ }^{1} \cdot$ Mari Miki $^{1}$ Shu-Ping Hui ${ }^{2} \cdot$ Rojeet Shrestha $^{2} \cdot K^{\prime}$ Kenji Yoshimura ${ }^{1} \cdot K_{\text {Kazuyuki Tsujino }}{ }^{~}$. \\ Hiroyuki Kagawa ${ }^{1} \cdot$ Yohei Oshitani $^{1} \cdot$ Hiroshi Kida $^{1} \cdot$ Ryoji Maekura $^{1,3} \cdot$ Kenji Kangawa $^{4}$
}

Received: 6 July 2019 / Accepted: 19 September 2019 / Published online: 8 October 2019

(c) The Physiological Society of Japan and Springer Japan KK, part of Springer Nature 2019

\begin{abstract}
The aim of this study was to investigate the effect of activated ghrelin with dietary octanoic acids or medium-chain triglyceride (MCT) administration to underweight patient with chronic obstructive pulmonary disease (COPD). Eleven severe and very severe COPD patients received a 5-day treatment with edible MCT. Sequentially, 10 patients received a 3-week combination treatment with MCT and intravenous acyl ghrelin. Five-day MCT treatment increased endogenous acyl ghrelin $(p=0.0049)$, but the total ghrelin level was unchanged. MCT-ghrelin combination treatment improved the peak oxygen uptake $(p=0.0120)$ during whole treatment course. This effect was attributed to the resultant improvements in cardiac function by $\mathrm{O}_{2}$ pulse, and to the difference between inspired and expired oxygen concentration rather than minute ventilation. Addition of dietary MCT to ghrelin treatment improved the aerobic capacity of underweight COPD patients, likely by mechanisms of increased $\mathrm{O}_{2}$ delivery through improvements in primary cardiocirculatory and muscular crosstalk.
\end{abstract}

Keywords Acylation $\cdot$ Circulation $\cdot$ Exercise $\cdot$ Underweight $\cdot$ Oxygen $\cdot$ Muscle

$\begin{array}{ll}\text { Abbreviations } \\ \text { COPD } & \text { Chronic obstructive pulmonary disease } \\ \text { CPET } & \text { Cardiopulmonary exercise testing } \\ \Delta F_{\mathrm{O}_{2}} & \begin{array}{l}\text { Inspired oxygen concentration }\left(\mathrm{Fi}_{\mathrm{O}_{2}}\right) \text { minus } \\ \text { expired oxygen concentration }\left(\mathrm{Fe}_{\mathrm{O}_{2}}\right)\end{array} \\ \text { FA } & \text { Fatty acid } \\ \text { MCT } & \text { Medium-chain triglyceride } \\ \text { MEP } & \text { Maximal expiratory pressure } \\ \text { MIP } & \text { Maximal inspiratory pressure } \\ \mathrm{O}_{2} \text { pulse } & V_{\mathrm{O}_{2}}^{\prime} / \text { HR } \\ \text { SGRQ } & \mathrm{St}^{2} \text { George Respiratory Questionnaire }\end{array}$

Keisuke Miki

miki.keisuke.pu@mail.hosp.go.jp

1 Department of Respiratory Medicine, National Hospital Organization Osaka Toneyama Medical Center, 5-1-1 Toneyama, Toyonaka, Osaka 560-8552, Japan

2 Faculty of Health Sciences, Hokkaido University, Kita 12, Nishi-5, Sapporo, Japan

3 Graduate School of Health Care Sciences, Jikei Institute, Miyahara 1-2-8, Yodogawa-ku, Osaka, Japan

4 National Cerebral and Cardiovascular Center Research Institute, 6-1, Kishibeshinmachi, Suita, Japan

$\begin{array}{ll}V_{\mathrm{D}} / V_{\mathrm{T}} & \text { Physiologic dead space/tidal volume-ratio } \\ V_{\mathrm{E}}^{\prime} & \text { Minute ventilation } \\ V_{\mathrm{O}_{2}}^{\prime} & \text { Oxygen uptake } \\ V_{\mathrm{T}} & \text { Tidal volume }\end{array}$

\section{Introduction}

Reduced aerobic capacity is a hallmark that has been related with the prognosis of patients with advanced stage of cachectic chronic obstructive pulmonary disease (COPD) $[1,2]$. Currently, COPD is the third leading cause of death globally [3]. Treatment strategies that target impaired aerobic function have been attempted to reduce the mortality, but further developments are necessary.

Gut-brain crosstalk has been attracting attention [4]. Ghrelin [5], which was first discovered from the stomach by Kojima and Kangawa et al. in 1999, is a 28 -amino acid peptide that has 2 forms, including des-acyl ghrelin and acyl ghrelin; the latter is considered the major active form. Soon after its discovery, ghrelin has been the only known peptide hormone with orexigenic effects [6], and has various physiologic effects [7-11]. Subsequently, we previously 
demonstrated that 3-week administration of intravenous acyl ghrelin alone [12] and with exercise training [13, 14] improved the impaired aerobic capacity, and symptoms of underweight patients with COPD.

Given that des-acyl ghrelin is converted to acyl ghrelin by an $n$-octanoyl modification at serine [15], and that this modification displays a higher affinity for octanoic acid C8:0 than for C6:0 and C10:0 [16], dietary octanoic acid C8:0 may be presumed to produce activated ghrelin. Notably, El Khoury et al. reported that the levels of acyl ghrelin increased soon after consumption of high-fat, low-carbohydrate meals [17], which are closely associated with a ketogenic diet. More recently, Kawai et al. reported that medium-chain triglyceride (MCT) supplementation increased the acyl ghrelin levels in patients with anorexia nervosa [18]. Dietary MCT, especially octanoic acid C8:0 might upregulate endogenous acyl ghrelin production. Furthermore, the combined effects of dietary octanoic acid C8:0 and intravenous acyl ghrelin administration might benefit the underweight COPD patient.

The present study aimed to investigate the mechanism, safety, and response to short-term administration of dietary octanoic acid C8:0 alone and a combination therapy with dietary octanoic acid C8:0 and intravenous ghrelin.

\section{Materials and methods}

\section{Study design and patients}

In this phase II, open-label clinical trial, underweight patients with severe and very severe COPD received a 5-day treatment with octanoic acid: C8:0 alone, followed by a 3-week combination treatment with octanoic acid: C8:0 and intravenous acyl ghrelin. Recruitment of patients was performed at a single center in the National Hospital Organization (NHO) Osaka Toneyama Medical Center, in Japan from October 2014 to March 2018. The study was conducted according to the Declaration of Helsinki and the Good Clinical Practice guidelines, and it was approved by the ethics committees of NHO Osaka Toneyama Medical Center (approval number, 1424). All patients gave written informed consent.

The inclusion criteria were as follows: (1) severe to very severe COPD [forced expiratory volume in one second $\left(\mathrm{FEV}_{1}\right) \%<70 \%$, and $\% \mathrm{FEV}_{1}<50 \%$ ]; (2) underweight, which was defined as a body mass index (BMI) of $<21 \mathrm{~kg} /$ $\left.\mathrm{m}^{2}\right)$; (3) in stable condition and able to tolerate cardiopulmonary exercise testing (CPET) to ensure adequate evaluation; (4) age between 20 and 85 years; and (5) provision of a signed agreement to participate in this study. Participants were excluded for any of the following reasons: (1) malignant tumors; (2) active infection; (3) severe heart disease; (4) hepatic failure (i.e., serum levels of aspartate transaminase and alanine transaminase greater than twice the upper limits of normal); (5) renal failure (i.e., serum creatinine concentration $\geq 2.0 \mathrm{mg} / \mathrm{dL}$ ); (6) asthma or positive airway reversibility test; (7) pregnant or possibly pregnant; (8) on therapy for diabetes mellitus or glycated hemoglobin $>7.0$; (9) change in the drug regimen within 1 month before participation in this study; and (10) judged by the physician to be inadequate to participate in this study.

This study was registered with the University Hospital Medical Information Network (UMIN) in Japan: https:// upload.umin.ac.jp/cgi-open-bin/icdr_e/ctr_view.cgi?recpt no=R000017686, number: UMIN000015222.

\section{Interventions}

Five grams of edible oil with octanoic acid C8 (The Nisshin OilliO Group, Ltd., Tokyo, Japan) were administered orally, twice daily, during breakfast and lunch, for 26 days (Fig. 1).

The preparation of human ghrelin was performed with the support from the National Cerebral and Cardiovascular Center Research Institute of Japan, as described previously [14]. Five-day treatment with MCT alone, followed by 3-week MCT-ghrelin combination treatment, were administered (Fig. 1). Synthetic human acyl ghrelin ( $2 \mu \mathrm{g} / \mathrm{kg}$, dissolved in $10 \mathrm{~mL}$ of sterile saline) was administered intravenously for $30 \mathrm{~min}$ twice daily, before breakfast and before lunch; the dose was determined based on previous data [19].

The continuation therapy with MCT intake as a posttherapy or the exercise training was not performed in the present study.

\section{Evaluation}

Pre-treatment measurements before 5-day MCT alone, and 3-week MCT-ghrelin combination treatment were performed at "pre-treatment" as shown in Fig. 1. Effects of 5-day MCT alone were examined once, after 5-day MCT. Effects of 3-week MCT-ghrelin combination treatment were examined twice, at week 3 and week 7 (i.e., 4 weeks after 3 -week combination treatment).

Pulmonary function tests were done, as previously described [20, 21]. Symptom-limited exercise tests were conducted on an electrically-braked cycle ergometer (CV1000SS, Lode, Groningen, The Netherlands), using a CPET system (Marquette CASE series T 2001, GE Healthcare, Tokyo, Japan; Aero monitor AE310S, Minato Medical Science Co., Ltd, Osaka, Japan), as previously described [21]. Incremental exercise testing, which comprised 2-min increments to $10 \mathrm{~W}$, was performed until patient exhaustion without encouragement, especially during exercise. All patients were asked to keep a cycle ergometer speed of about $60 \mathrm{rpm}$ while looking at the rpm meter. Minute ventilation $\left(V_{\mathrm{E}}^{\prime}\right)$, oxygen uptake $\left(V_{\mathrm{O}_{2}}^{\prime}\right)$, car- 
Fig. 1 Trial profile

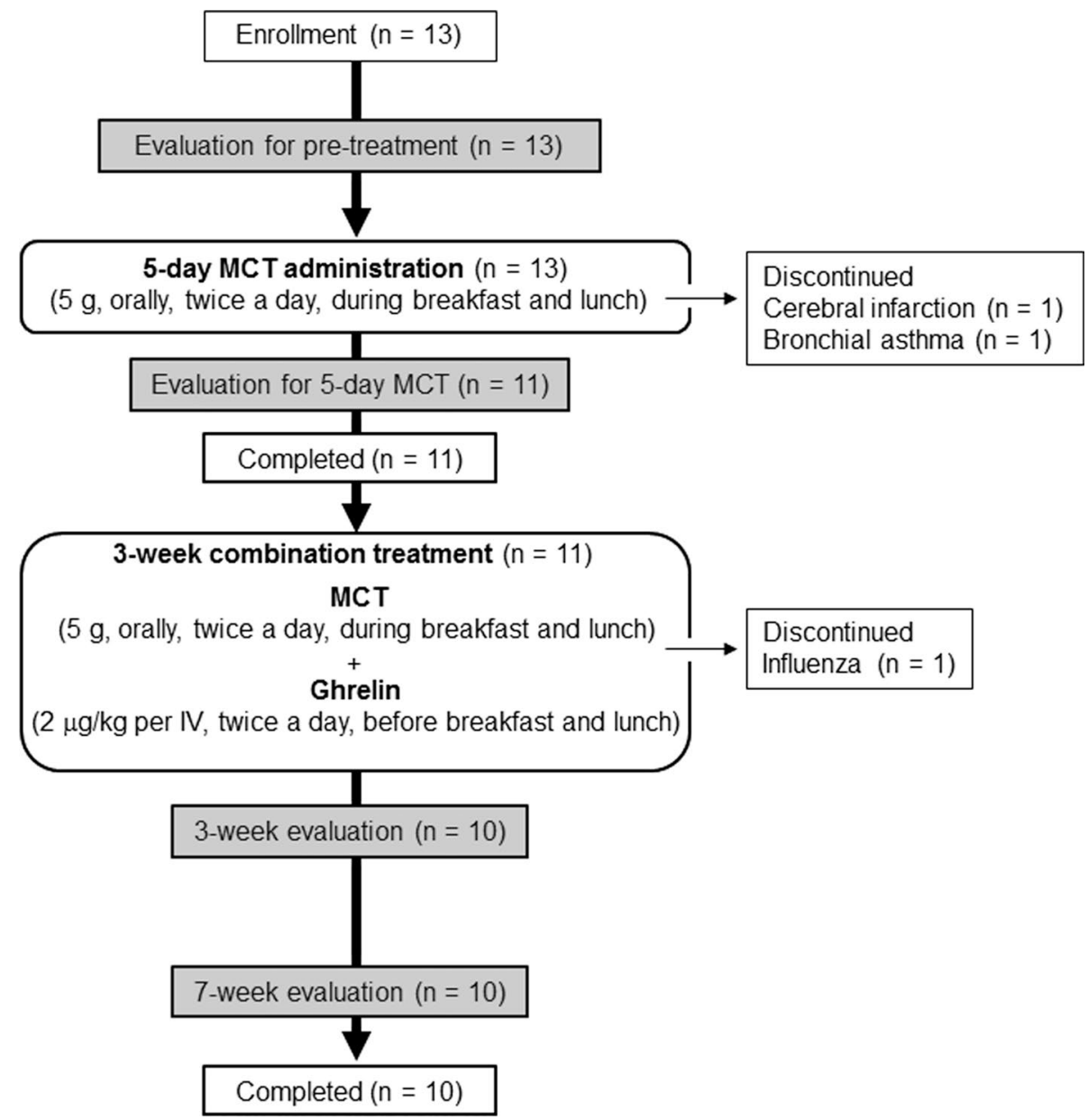

bon dioxide output $\left(V_{\mathrm{CO}_{2}}^{\prime}\right)$, breathing frequency $\left(f_{\mathrm{R}}\right)$, tidal volume $\left(V_{\mathrm{T}}\right)$, inspired oxygen concentration $\left(\mathrm{Fi}_{\mathrm{O}_{2}}\right)$, expired oxygen concentration $\left(\mathrm{Fe}_{\mathrm{O}_{2}}\right)$, physiologic dead space/tidal volume ratio $\left(V_{\mathrm{D}} / V_{\mathrm{T}}\right)$, and oxygen $\left(\mathrm{O}_{2}\right)$ pulse $\left[V_{\mathrm{O}_{2}}^{\prime} /\right.$ heart rate $(\mathrm{HR})]$, were measured breath-by-breath and were collected as 30-s average values at rest, during exercise at 2-min intervals, and at the end of exercise. $\mathrm{O}_{2}$ pulse, which is almost equivalent to the stroke volume, was measured as an indicator of exertional cardiac function, because cardiac output equals the product of stroke volume and HR. The severity of dyspnea and intensity of leg fatigue were evaluated by the modified Borg scale [22] at rest and during the last $15 \mathrm{~s}$ of each exercise stage and at the end of exercise. $\Delta \mathrm{F}_{\mathrm{O}_{2}}$ was calculated as the difference between $\mathrm{Fi}_{\mathrm{O}_{2}}$ and $\mathrm{Fe}_{\mathrm{O}_{2}}$. Dual-energy X-ray absorptiometry, 6-min walk distance test, assessment of food intake for 3 days, and the respiratory muscle strength by the maximal inspiratory and expiratory pressures (MIP and MEP, respectively) were performed as described previously [14].

The St. George Respiratory Questionnaire (SGRQ) was used to assess the respiratory health status of patients with COPD. In the present study, the validated Japanese version of the SGRQ was used [23, 24]. The SGRQ is a 50-item questionnaire that is divided into 3 domains: symptoms (i.e., assessment of the frequency and severity of respiratory symptoms); activity (i.e., assessment of the effects of breathlessness on mobility and physical activity); and impact (i.e., assessment of the social function and psychosocial disturbances of the disease). The items were scored on a scale of $0-100$, with higher scores reflecting worse respiratory health status. The minimum clinically important difference in the SGRQ was accepted as four units [25].

Blood sampling for plasma ghrelin levels was performed after $30 \mathrm{~min}$ of bed rest at 7:00 a.m., 10:00 a.m., 2:00 p.m., and 4:00 p.m. on the day of pre-treatment, the last day of the 5-day MCT treatment, and the last day of the 3-week MCT-ghrelin combination treatment. Blood sampling and measurements of plasma ghrelin were performed (Tosoh Co; Tokyo, Japan), as previously described [26]. Blood sampling for plasma free fatty acid (FA) 8:0 was performed at 10:00 a.m. on the day of pre-treatment, the last day of the 5-day MCT treatment, and the last day of the 3-week MCT-ghrelin combination treatment. Plasma free FA 8:0 was measured in the Faculty of Health Sciences, Hokkaido 
University, Japan, as previously described [27, 28]. Urinary norepinephrine level was measured by 24 -h collection (LSI Medience Corporation, Tokyo, Japan).

\section{Outcome measures}

\section{Efficacy}

The primary outcomes were changes in peak $V_{\mathrm{O}_{2}}^{\prime}$ and the SGRQ. The secondary outcomes included the changes in the following variables: $\mathrm{O}_{2}$ pulse, ventilatory equivalent for $\mathrm{O}_{2}$ $\left(V_{\mathrm{E}}^{\prime} / V_{\mathrm{O}_{2}}^{\prime}\right), V_{\mathrm{D}} / V_{\mathrm{T}}$, respiratory muscle strength, body weight, 6-min walk distance, food intake, circadian changes in the plasma acyl ghrelin level, and plasma free FA 8:0.

\section{Safety}

All participants who received at least 1 of the study treatments were included in the safety analyses.

\section{Sample size and statistical analysis}

At the time of the study design, the definition of a clinically important change after MCT-ghrelin combination treatment was unknown. Therefore, sample size calculation was performed based on the estimated effect of a 3-week ghrelin-pulmonary rehabilitation combination treatment on peak $V_{\mathrm{O}_{2}}^{\prime}$ obtained by CPET [13]. Using a two-tailed test and an $\alpha$ of 0.05 , a sample size of 7 was calculated to provide a $90 \%$ power to detect a mean peak $V_{\mathrm{O}_{2}}^{\prime}$ difference of $1.22 \mathrm{~mL} / \mathrm{kg} /$ $\mathrm{min}$, with an estimated standard deviation of $0.8 \mathrm{~mL} / \mathrm{kg} / \mathrm{min}$. To obtain 7 evaluable participants, the total sample size was set at 10 .

The effects of the 5-day MCT treatment were examined once (i.e., after 5 days). The effects of the 3-week MCT-ghrelin combination treatment were examined twice during the course, particularly after 3 weeks (i.e., week 3 ) and 4 weeks after its completion (i.e., week 7). The changes in each outcome before and after the 5-day MCT treatment were analyzed using the Wilcoxon signed rank test. The changes in each outcome during the treatment time course or sampling time course were analyzed using a linear mixed effect model, considering patients as the random effect and the treatment points or sampling points as the fixed effect. The least squares means Tukey's honestly significant difference test was used for the comparison of each variable among the treatment points. A $p$ value of $<0.05$ was considered significant. Statistical analyses were performed using JMP software, version 11 (SAS Institute Inc., Cary, NC, USA).

\section{Results}

Of the 13 enrolled patients, 11 completed the 5-day treatment with MCT alone and 10 completed the entire study protocol (Fig. 1). Two patients dropped out during the 5-day treatment with MCT alone, and 1 patient dropped out during the MCT-ghrelin combination treatment. Therefore, to ensure efficacy evaluation using per protocol analysis, 11 patients were included in the analyses of the 5-day MCT treatment and 10 were included in the analyses of the MCT-ghrelin combination treatment. Table 1 shows the patients' baseline characteristics.

Table 1 Baseline characteristics of the patients $(N=11)$

\begin{tabular}{|c|c|}
\hline Age, years & $71.3(4.8), 64-78$ \\
\hline Sex, male/female (number) & $10 / 1$ \\
\hline BMI, $\mathrm{kg} / \mathrm{m}^{2}$ & $17.4(1.9), 13.9-20.9$ \\
\hline Cigarette smoking, pack years & 54.6 (24.7), $24.5-98$ \\
\hline GOLD, III/IV, number & $6 / 5$ \\
\hline \multicolumn{2}{|l|}{ Pulmonary function test } \\
\hline $\mathrm{FEV}_{1}, \mathrm{~L}$ & $0.82(0.25), 0.41-1.29$ \\
\hline$\% \mathrm{FEV}_{1}, \%$ predicted & $32.2(9.4), 16.8-48.2$ \\
\hline $\mathrm{FEV}_{1} / \mathrm{FVC}, \%$ & $34.3(6.6), 22.9-46.0$ \\
\hline $\mathrm{VC}, \mathrm{L}$ & $2.47(0.57), 1.83-3.39$ \\
\hline$\% \mathrm{VC}, \%$ & $78.3(15.7), 55.6-100.5$ \\
\hline \multicolumn{2}{|c|}{ Incremental work rate exercise testing } \\
\hline \multicolumn{2}{|l|}{ At peak exercise } \\
\hline Dyspnea, Borg scale & $5.8(1.2), 4-7$ \\
\hline$V_{\mathrm{O}_{2}}^{\prime}, \mathrm{mL} / \mathrm{min} / \mathrm{kg}$ & $10.6(2.6), 6.9-14.1$ \\
\hline \multicolumn{2}{|l|}{ SGRQ } \\
\hline Total score & $55.6(17.7), 26.8-79.9$ \\
\hline Symptoms score & 67.5 (20.6), 19.1-92.0 \\
\hline Activity score & $70.1(13.1), 54.4-92.5$ \\
\hline Impacts score & 40.3 (22.4), 4.9-73.4 \\
\hline \multicolumn{2}{|l|}{ Medications (number) } \\
\hline LAMA & 11 \\
\hline LABA & 9 \\
\hline ICS & 6 \\
\hline Triple inhalation therapy & 5 \\
\hline Methylxanthines & 0 \\
\hline
\end{tabular}

Data are presented as mean (SD) and as the minimum and maximum values, unless otherwise stated

$B M I$ body mass index, $F E V_{l}$ forced expiratory volume in one second, $F V C$ forced vital capacity, GOLD global initiative for chronic obstructive lung disease, ICS inhaled corticosteroids, $L A B A$ long-acting $\beta_{2}$-agonist, LAMA long-acting muscarinic antagonist, $S G R Q$ St. George Respiratory Questionnaire; Triple inhalation; a combination of LAMA, LABA, and ICS, $V C$ vital capacity, $V_{O_{2}}^{\prime}$ oxygen uptake

Medications are presented separately 


\section{Response to 5-day MCT treatment}

During the whole period of 5-day MCT and 3-week MCT-ghrelin combination treatment, acyl ghrelin levels were analyzed based on the sampling time course among the treatments (Fig. 2a). Given that, at 4:00 PM, the acyl ghrelin level was at its highest in the sampling time course analysis by a linear mixed effect model $(p=0.0003$, Fig. 2a), acyl ghrelin levels after 5-day treatment with MCT alone were analyzed, focusing on that point (Table 2, Fig. 2b). After 5-day treatment with MCT alone, the plasma free FA 8:0, which was obtained at 10:00 a.m., increased significantly, compared with the pre-treatment levels $(p=0.0010$, Table 2$)$ and during the treatment time course $(p<0.0001$, Fig. 2 b); at 4 h (i.e., 4:00 p.m.) after the daily intake of $10 \mathrm{~g}$ of MCT during the sampling period, the acyl ghrelin level was increased, compared with the pre-treatment level ( $p=0.0049$, Table 2); and at that same time, the des-acyl ghrelin level decreased significantly, compared with the pre-treatment level $(p=0.0098$, Table 2$)$.

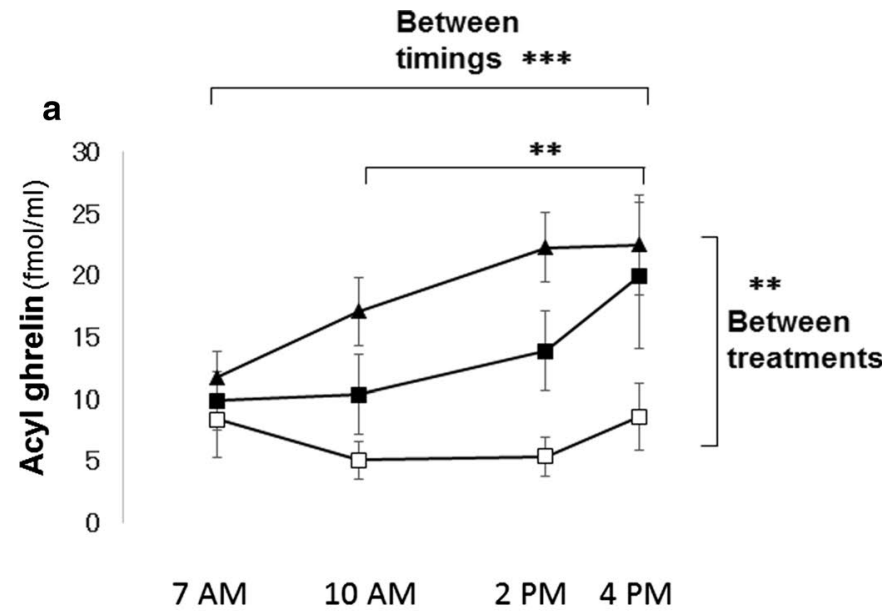

Fig. 2 a Time course of plasma acyl ghrelin after 5-day MCT or 3-week MCT-ghrelin combination treatment. Changes in plasma acyl ghrelin were evaluated at 7:00 a.m., 10:00 a.m., 2:00 p.m., and 4:00 p.m. among treatments. Significant changes in the treatments $(p=0.0097)$, and in the sampling timings $(p=0.0003)$ are confirmed by a linear mixed effect model. ${ }^{* *} p<0.01,{ }^{* * *} p<0.001$ between different timings or treatments, using the least squares means Tukey's honestly significant difference test. Open square, pre-treatment; closed square, after 5-day intake of MCT with octanoic acid C 8:0 alone; closed triangle, after 3-week MCT-ghrelin combination treatment. b Comparison of the changes in the plasma acyl ghrelin and
Table 2 Responses to dietary medium-chain triglycerides for 5 days $(N=11)$

\begin{tabular}{lccc}
\hline & Before $(n=11)$ & After $(n=11)$ & $p$ value \\
\hline $\begin{array}{l}\text { Free fatty acids 8:0, } \\
\mu \mathrm{moL} / \mathrm{L}\end{array}$ & $2.7(1.4)$ & $20.5(10.4)$ & 0.0010 \\
$\quad$ Acyl ghrelin, fmoL/mL & $8.6(9.1)$ & $20.0(19.7)$ & 0.0049 \\
Des-acyl ghrelin, fmoL/mL & $53.7(44.3)$ & $38.0(27.8)$ & 0.0098 \\
Total ghrelin, fmoL/mL & $62.3(53.1)$ & $57.9(45.9)$ & 0.3652 \\
\hline
\end{tabular}

Data are means (SD). Blood samples for the free fatty acids 8:0 in plasma were collected at 10:00 AM (2 h after the morning intake of $5 \mathrm{~g}$ of medium-chain triglycerides). Blood samples for acyl ghrelin and desacyl ghrelin were collected at 4:00 p.m. ( $4 \mathrm{~h}$ after the 12:00 p.m. intake of $5 \mathrm{~g}$ of medium-chain triglycerides, when the mean concentration of acyl ghrelin was the highest among the sampling periods. Change between before and after dietary MCT for 5 days by the Wilcoxon signed rank test

\section{Response to 3-week MCT-ghrelin combination treatment}

Body weight $(p=0.0003)$ and SGRQ symptoms $(p=0.0153)$ improved during the course of 3-week MCT-ghrelin combination treatment (Table 3).

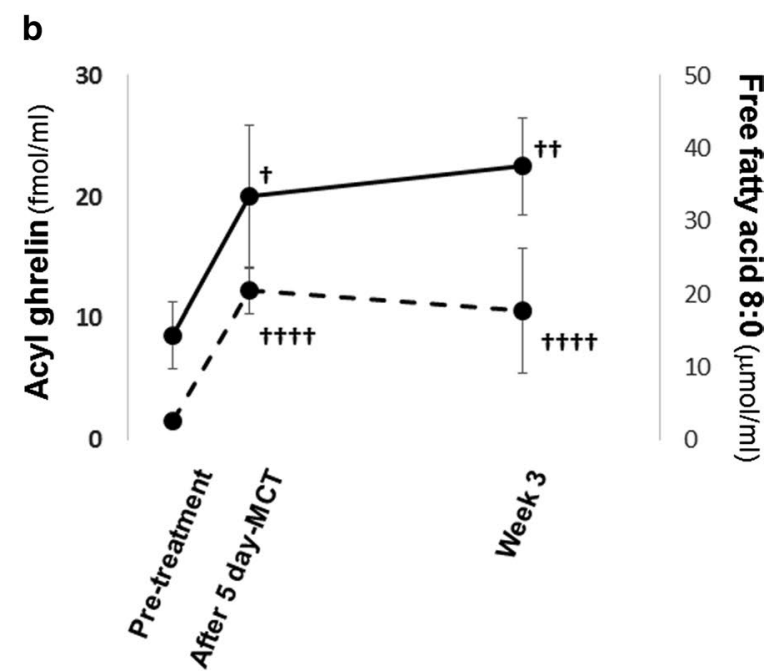

free FA 8:0 levels during the treatment course of a 5-day intake of MCT alone, followed by 3-week MCT-ghrelin combination treatment. Significant increases in the plasma levels of free FA 8:0, obtained at 10:00 a.m. $(p<0.0001)$ and acyl ghrelin $(p=0.0072)$, obtained at 4:00 p.m. are confirmed by a linear mixed effect model. ${ }^{\dagger} p<0.05,{ }^{\dagger \dagger} p<0.01,{ }^{\dagger \dagger \dagger} p<0.0001$, compared with the pre-treatment values, using the least squares means Tukey's honestly significant difference test. Continuous line, plasma acyl ghrelin; dashed line, free FA 8:0. Data are presented as means with standard error. $F A$ fatty acid, $M C T$ medium-chain triglyceride 
Table 3 Changes in the resting parameters after a 3 -week combination therapy with ghrelin and dietary mediumchain triglycerides $(N=10)$

\begin{tabular}{|c|c|c|c|c|}
\hline & Pre-treatment & At 3 weeks & At 7 weeks & $p$ value \\
\hline Body weight, $\mathrm{kg}$ & $46.1(7.7)$ & $47.5(7.2)^{* *}$ & $48.2(8.0)^{* * *}$ & 0.0003 \\
\hline BMI, $\mathrm{kg} / \mathrm{m}^{2}$ & $17.4(2.1)$ & $18.1(1.5)^{*}$ & $18.3(1.8)^{* * *}$ & 0.0008 \\
\hline Total lean mass, $\mathrm{kg}$ & $36.1(4.7)$ & $37.0(4.5)^{* *}$ & $35.9(4.6)^{\dagger \dagger}$ & 0.0011 \\
\hline Food intake, kcal/day & $1853(264)$ & $1962(318)$ & ND & 0.0360 \\
\hline $\mathrm{MEP}, \mathrm{cmH}_{2} \mathrm{O}$ & $99.7(26.2)$ & $106.9(34.7)$ & $102.0(38.4)$ & 0.5413 \\
\hline MIP, $\mathrm{cmH}_{2} 0$ & $-56.3(12.6)$ & $-63.7(14.2)$ & $-59.3(12.0)$ & 0.2058 \\
\hline $\begin{array}{l}\text { Urinary NE on } 24-\mathrm{h} \text { collec- } \\
\text { tion, } \mu \mathrm{g} / \text { day }\end{array}$ & $268.7(121.9)$ & $126.1(48.3)$ & ND & 0.0063 \\
\hline \multicolumn{5}{|l|}{ Pulmonary function test } \\
\hline $\mathrm{FEV}_{1}, \mathrm{~L}$ & $0.85(0.24)$ & $0.87(0.20)$ & $0.86(0.21)$ & 0.8963 \\
\hline $\mathrm{FEV}_{1} / \mathrm{FVC}, \%$ & $34.2(6.9)$ & $33.0(5.9)$ & $33.0(6.4)$ & 0.5492 \\
\hline$\% \mathrm{FEV}_{1}, \%$ & $33.4(9.0)$ & $34.4(7.6)$ & $34.2(8.1)$ & 0.7830 \\
\hline $\mathrm{VC}, \mathrm{L}$ & $2.53(0.56)$ & $2.60(0.51)$ & $2.58(0.45)$ & 0.8298 \\
\hline$\% \mathrm{VC}, \%$ & $80.6(14.5)$ & $83.9(12.1)$ & $83.6(10.8)$ & 0.6316 \\
\hline \multicolumn{5}{|l|}{ SGRQ } \\
\hline Symptoms & $66.4(21.4)$ & $50.9(22.3)^{*}$ & $56.2(17.4)$ & 0.0153 \\
\hline Activity & $67.9(11.4)$ & $70.6(12.5)$ & $67.8(17.3)$ & 0.6497 \\
\hline Impacts & $40.3(21.1)$ & $36.8(16.6)$ & 44.4 (25.9) & 0.1197 \\
\hline Total & $53.2(16.6)$ & $49.8(14.2)$ & $53.5(21.0)$ & 0.3713 \\
\hline
\end{tabular}

Data are presented as means (SD)

The resultant $p$ values are represented. The variables were tested by a linear mixed effect model

$F E V_{l}$ forced expiratory volume in one second, $F V C$ forced vital capacity, $M E P$ maximal expiratory pressure, $M I P$ maximal inspiratory pressure, $N E$ norepinephrine level, $S G R Q$ St. George Respiratory Questionnaire, $V C$ vital capacity

${ }^{*} p<0.05,{ }^{* *} p<0.01,{ }^{* * *} p<0.001$ by the least squares means Tukey's honestly significant difference test, compared with pre-treatment values

${ }^{\dagger} p<0.05,{ }^{\dagger \dagger} p<0.01$ by the least squares means Tukey's honestly significant difference test, compared with the values at 3 weeks
As shown in Table 4 and Fig. 3, during the time course of MCT-ghrelin combination treatment, there were significant improvements at peak exercise in $V_{\mathrm{O}_{2}}^{\prime}(p=0.0120), V_{\mathrm{E}}^{\prime}$ $(p=0.0141)$, and $\Delta \mathrm{F}_{\mathrm{O}_{2}}$ (i.e., inspired $\mathrm{O}_{2}$ concentration minus expired $\mathrm{O}_{2}$ concentration) $(p=0.0349)$ with a linear mixed effect model that estimated the effects during whole treatment course. When comparing each parameter among the treatment points, a significant improvement was observed in the $V_{\mathrm{E}}^{\prime}$ at week 3, followed by improvements in $V_{\mathrm{O}_{2}}^{\prime}, \Delta \mathrm{F}_{\mathrm{O}_{2}}$, $V_{\mathrm{E}}^{\prime} / V_{\mathrm{O}_{2}}^{\prime}$, and $\mathrm{O}_{2}$ pulse at week 7. At isotime exercise, there was a significant improvement in leg discomfort $(p=0.0136)$ rather than in dyspnea $(p=0.0556)$ during the time course.

\section{Safety}

Passage of loose stools was not reported during the 5-day MCT treatment, but it was observed in 5 of 13 (38\%) patients during the 3-week MCT-ghrelin combination treatment (Table 5). However, diarrhea was not reported. No treatment-related serious events were reported in the present study. As shown in Fig. 1, 3 patients discontinued the treatment because of influenza infection $(n=1)$, bronchial asthma necessitating changes in the drug regimen immediately after the enrollment in the present trial $(n=1)$, and cerebral infarction on the 3rd day of the 5-day treatment with MCT alone $(n=1)$. The latter was judged by the efficacy and safety committee as not causally related with the MCT treatment, considering the presence of multiple old lacunar infarcts in the brainstem and in both basal nuclei on brain magnetic resonance imaging and carotid artery atherosclerotic plaque on carotid ultrasound and the safety of MCT as a food product was not doubtful, because it is also contained in milk and coconut oil.

\section{Discussion}

The main findings of this study on underweight COPD patients were (1) 5-day treatment with MCT alone immediately increased the plasma acyl ghrelin level, but it did not affect the total ghrelin level and (2) MCT-ghrelin combination therapy improved the SGRQ symptom score and peak $V_{\mathrm{O}_{2}}^{\prime}$ during incremental exercise. 
Table 4 Changes in exercise testing after a 3 -week combination therapy with ghrelin and dietary mediumchain triglycerides $(N=10)$

\begin{tabular}{lllll}
\hline & Pre-treatment & At 3 weeks & At 7 weeks & $p$ value \\
\hline 6-MWD, m & $304(104)$ & $347(99)^{*}$ & $351(107)^{*}$ & 0.0107 \\
CPET, at peak exercise & & & \\
$V_{\mathrm{O}_{2}}^{\prime}, \mathrm{mL} / \mathrm{min} / \mathrm{kg}$ & $10.9(2.6)$ & $11.5(2.2)$ & $11.8(2.2)$ & 0.1873 \\
$V_{\mathrm{O}}^{\prime}, \mathrm{mL} / \mathrm{min}$ & $495.1(109.6)$ & $543.1(122.8)$ & $562.3(109.1)^{*}$ & 0.0120 \\
Endurance time, s & $333(157)$ & $400(112)^{* *}$ & $389(107)^{*}$ & 0.0065 \\
Dyspnea (Borg scale) & $5.7(1.2)$ & $6.5(1.8)$ & $5.8(2.0)$ & 0.1078 \\
Leg discomfort (Borg scale) & $4.8(2.6)$ & $4.1(2.3)$ & $3.9(2.6)$ & 0.3907 \\
$V_{\mathrm{T}}, \mathrm{L}$ & $0.86(0.17)$ & $0.92(0.14)$ & $0.95(0.14)$ & 0.0838 \\
$f_{\mathrm{R}}$, breaths/min & $30(7)$ & $30(6)$ & $28(6)$ & 0.0442 \\
$V_{\mathrm{E}}^{\prime}, \mathrm{L} / \mathrm{min}$ & $24.9(6.1)$ & $28.0(7.7)^{*}$ & $26.6(7.3)$ & 0.0141 \\
$\Delta \mathrm{F}_{\mathrm{O}_{2}}, \%$ & $2.48(0.40)$ & $2.41(0.26)$ & $2.62(0.34)^{\dagger}$ & 0.0349 \\
$V_{\mathrm{D}} / V_{\mathrm{T}}$ & $0.43(0.06)$ & $0.44(0.03)$ & $0.43(0.05)$ & 0.6099 \\
$V_{\mathrm{E}}^{\prime} / V_{\mathrm{O}_{2}}^{\prime}$ & $50.7(7.9)$ & $51.4(5.4)$ & $47.1(6.8)^{* \dagger \dagger}$ & 0.0051 \\
$V_{\mathrm{E}}^{\prime} / V_{\mathrm{CO}}^{\prime}$ & $50.8(6.9)$ & $49.1(4.9)$ & $47.7(6.3)$ & 0.0824 \\
$R$ & $1.00(0.07)$ & $1.05(0.09)^{* *}$ & $0.99(0.08)^{\dagger \dagger \dagger}$ & 0.0004 \\
$\mathrm{HR}_{2}$, beats/min & $114(20)$ & $121(20)^{*}$ & $116(19)$ & 0.0296 \\
$\mathrm{O}_{2}$ pulse, mL/beats & $4.3(0.5)$ & $4.5(0.6)$ & $4.9(0.8)^{* * \dagger}$ & 0.0043 \\
$\mathrm{SpO}_{2}, \%$ & $90.7(5.7)$ & $89.5(3.7)$ & $91.0(4.0)$ & 0.3575 \\
\hline
\end{tabular}

Two patients performed CPET while breathing the same $\mathrm{Fi}_{\mathrm{O}_{2}}$ of $24 \%$ pre-treatment and post-treatment. Data are presented as means (SD)

The resultant $p$ values are represented. The variables were tested by a linear mixed effect model

CPET cardiopulmonary exercise testing, $\Delta F_{O_{2}}$ : inspired oxygen concentration $\left(\mathrm{FiO}_{2}\right)$ minus expired oxygen concentration $\left(\mathrm{FeO}_{2}\right), f_{R}$ breathing frequency, $H R$ heart rate, 6-MWD 6-min walk distance, $R$ respiratory quotient, $\mathrm{SpO}_{2}$ percutaneous oxygen saturation, $V_{D} / V_{T}$ physiologic dead space/tidal volume ratio, $V_{E}{ }_{E}$ minute ventilation, $V_{\mathrm{CO}_{2}}^{\prime}$ carbon dioxide output, $V_{\mathrm{O}_{2}}^{\prime}$ oxygen uptake, $\mathrm{O}_{2}$ pulse $V_{\mathrm{O}_{2}}^{\prime} / \mathrm{HR}, V_{T}$ tidal volume

${ }^{*} p<0.05,{ }^{* *} p<0.01$, by the least squares means Tukey's honestly significant difference test, compared with pre-treatment values

${ }^{\dagger} p<0.05,{ }^{\dagger \dagger} p<0.01,{ }^{\dagger \dagger} p<0.001$, by the least squares means Tukey’s honestly significant difference test, compared with the values at 3 weeks
Given the observed change from des-acyl ghrelin to acyl ghrelin after a 5-day treatment with MCT alone, edible MCT may be of therapeutic relevance in cachectic COPD. Ghrelin secretion is regulated by various factors, including exercise $[29,30]$ and meal $[6,31]$, both of which affect the daily condition of patients with advanced COPD. In such patients, although elevated ghrelin levels were reported [32], intense acute exercise might suppress the level of acyl ghrelin (i.e., active form) in everyday life. Furthermore, plasma ghrelin levels have been demonstrated to increase before each meal, but to fall to trough levels after every meal [31]. Based on the fact that octanoic acid C8:0 was primarily connected with des-acyl ghrelin [5], dietary octanoic acid may be necessary for the acyl modification of ghrelin [33]. This response was confirmed by the results of the present study, which showed that a 5-day MCT treatment did not change the total ghrelin level but increased the acyl ghrelin/des-acyl ghrelin ratio (Table 2). Moreover, in the present study, the increased plasma acyl ghrelin level was confirmed during the daytime after 3-week MCT-ghrelin combination treatment (Fig. 2a). It is highly possible that the resultant increase of plasma acyl ghrelin during the daytime was induced by MCT treatment alone. The reasons are as follows: the acyl ghrelin peaked and disappeared rapidly from plasma with an elimination half-life of 9-13 min after the intravenous ghrelin administration [34, 35], and each sampling time for plasma acyl ghrelin was more than $2 \mathrm{~h}$ from the intravenous ghrelin treatment in the present study. An MCT-based ketogenic diet has been increasingly explored in a number of neurologic diseases and some cancers $[36,37]$. At the very least, the ketogenic diet, especially via octanoic acid, may provide various benefits, including an alternative energy supply, for cachectic COPD patients, because it can maintain high levels of acyl ghrelin at all times by suppressing the decrease in the levels after each meal or during intolerable acute exercise.

Cardiocirculatory and muscular crosstalk, which is a mechanism that is related with $\mathrm{O}_{2}$ delivery and utilization, has been increasingly described in patients with cardiopulmonary disease, although its degree may differ among patients. In COPD-heart failure overlap patients, the index of cardiac output and the index of peripheral blood flow to a certain exercise load were impaired [38-40]. In other 
Fig. 3 Changes in the physiological variables after a 3-week combination treatment with octanoic acid C8:0 and ghrelin. Each physiological variable was obtained at peak exercise during incremental cardiopulmonary exercise testing. The changes in each variable during the treatment course are analyzed by a linear mixed effect model. Compared with pre-treatment, ${ }^{*} p<0.05,{ }^{* *} p<0.01$ and compared with the values at week $3,{ }^{\dagger} p<0.05,{ }^{\dagger \dagger} p<0.01$ using the least squares means Tukey's honestly significant difference test. $\Delta F_{O_{2}}$ inspired $\mathrm{O}_{2}$ concentration minus expired $\mathrm{O}_{2}$ concentration, $M C T$ medium-chain triglyceride; $\mathrm{O}_{2}$ pulse $V_{\mathrm{O}_{2}}^{\prime}$ /heart rate, $V_{E}^{\prime}$ minute ventilation, $V_{O_{2}}^{\prime}$ oxygen uptake

words, it might be difficult to improve anaerobic capacity of advanced COPD patients by increasing ventilation alone. In fact, a previous study showed that in $50 \%$ of patients with advanced COPD, the reduction of peak $V_{\mathrm{O}_{2}}^{\prime}$ with pulmonary rehabilitation was due to a decrease in $\Delta \mathrm{F}_{\mathrm{O}_{2}}$, even if the exercise time was prolonged [41]. In the present study, MCT-ghrelin combination therapy improved the symptoms and anaerobic capacity of patients with severe and very severe COPD along with weight gain. In a previous study $[13,14]$, we reported that combination therapy with exercise training and ghrelin improved the anaerobic capacity of COPD patients without weight gain, whose baseline peak $V_{\mathrm{O}_{2}}^{\prime}$ was slightly higher than that of the patients in the present study. In both trials, the mean changes in peak $V_{\mathrm{O}_{2}}^{\prime}$ and 6-min walk distance (6-MWD) from the pre-treatment values were approximately similar. In the present study, however, improvements in ventilatory condition, such as expiratory respiratory pressure, $V_{\mathrm{T}}$, and $V_{\mathrm{D}} / V_{\mathrm{T}}$, were not obtained, which might be due to the fact that exercise training was not conducted in the present study. Notably, at 4 weeks after the completion of the 3-week combination therapy, i.e., week 7 , improvements in peak $V_{\mathrm{O}_{2}}^{\prime}$ and 6-MWD were obtained (Fig. 3 and Table 4). Because MCT continuation therapy was not performed after the present study, the effects of the activated ghrelin including MCT intake might remain large at 4 weeks after the completion of the 3 -week combination therapy, although other evaluation points after week 7 to confirm whether the obtained effects return to the pretreatment level were not investigated in the present study. At week 7 in the present study, the resultant increase in the $\mathrm{O}_{2}$ pulse and $\Delta \mathrm{F}_{\mathrm{O}_{2}}$ might explain the differences in the results between the studies and may be the primary reason for the improved aerobic capacity, based on the following mechanism. Due to the direct effect of ghrelin on vasodilation [42] and cardiac output [8-10], the resultant vasodilatory effect and improvement in cardiac function, which was assessed by the $\mathrm{O}_{2}$ pulse, might increase muscle blood flow, followed by an increase in $\Delta \mathrm{Fo}_{2}$. These implied that the absorption ability for $\mathrm{O}_{2}$ was improved by enhancing the direct effects of ghrelin on cardiocirculatory function.
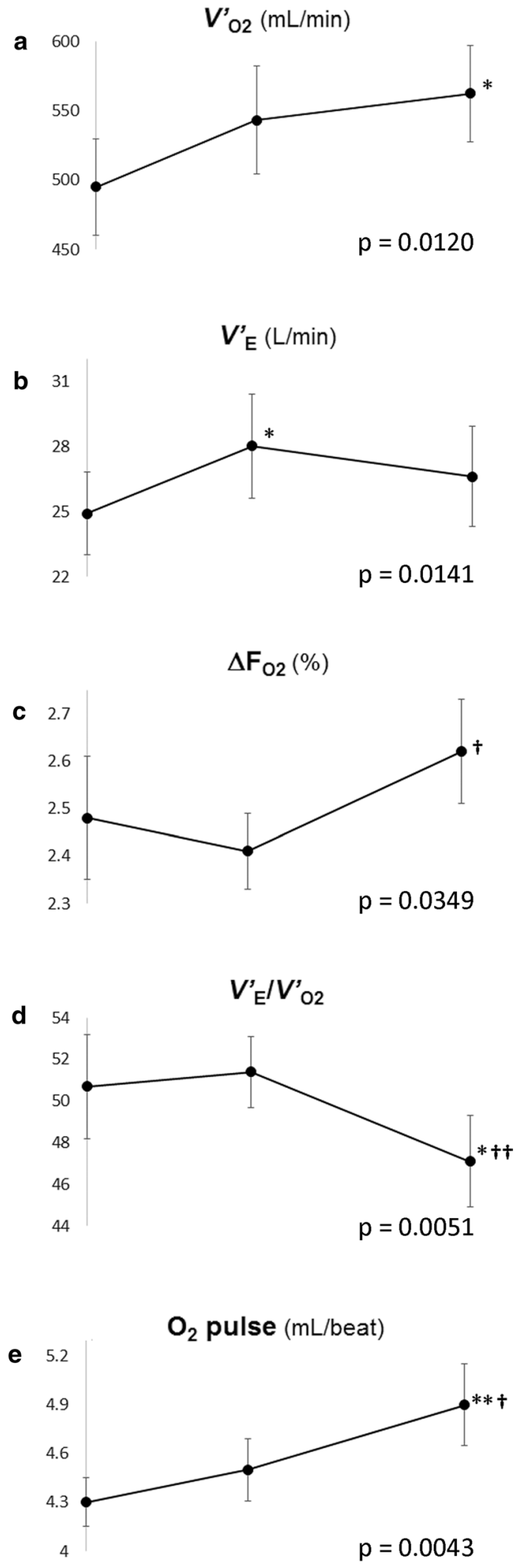

Pre-treatment Week $3 \quad$ Week 7 
Table 5 Adverse events $(N=13)$

\begin{tabular}{lcl}
\hline Adverse events & $\begin{array}{c}\text { 5-day MCT } \\
\text { treatment }\end{array}$ & $\begin{array}{l}\text { 3-week ghrelin and MCT } \\
\text { combination treatment }\end{array}$ \\
\hline Unclearly related with the trial intervention & 5 \\
Loose stools & 1 \\
Stomach rumbling & 2 \\
Feeling of being warm & 2 \\
Slight elevation of CRP & 1 \\
Slight liver dysfunction & \\
Considered not to be related with the trial intervention \\
Bronchial asthma & 1 & \\
Cerebral infarction & 1 & 1 \\
Influenza infection & & \\
\hline
\end{tabular}

Data are presented as the number of patients

$C R P$ C-reactive protein, $M C T$ medium-chain triglyceride

Considering that $V_{\mathrm{O}_{2}}^{\prime}$ is calculated using the product of $V_{\mathrm{E}}^{\prime}$ and $\Delta \mathrm{Fo}_{2}$ and the present findings of slightly reduced $V_{\mathrm{E}}^{\prime}$ at week 7 than at week 3 , the improvement in peak $V_{\mathrm{O}_{2}}^{\prime}$ may be attributed to an improved $\mathrm{O}_{2}$ absorption ability by the increased blood flow through cardiocirculatory and muscular crosstalk. In addition, leg discomfort, rather than dyspnea, was improved at isotime after the combination therapy. Furthermore, the slight decrease in $V_{\mathrm{E}}^{\prime}$ at week 7 might imply a decreased ventilatory demand; as a result, the $V_{\mathrm{E}^{\prime}}^{\prime} / V_{\mathrm{O}_{2}}^{\prime}$ was reduced. Taken together, treatment strategies that target activated ghrelin to improve the $\mathrm{O}_{2}$ absorption ability from cardiac and/or skeletal muscle function may prove beneficial for the impaired anaerobic condition in cachectic COPD.

Developing a treatment strategy including exercise training in cachectic patients with COPD is an important issue. Anamorelin, a novel ghrelin-receptor agonist, is now attracting attention as a treatment option given orally to patients with cancer anorexia and cachexia [43]. Evaluating the sequencing of exercise training and activated ghrelin therapy might be important. When exercise training is performed with activated ghrelin treatment including anamorelin, even if the intensity of the frequency of exercise training is suitable for each patient, exercise training should be added as a post-therapy after sufficient effects are obtained by the activated ghrelin treatment, because, even if exercise training is not added, activated ghrelin treatment may provide sufficient pharmacological effects, as in the present study, and, in the previous study [14], the number of days of exercise training in the ghrelin-exercise training group was negatively correlated with the increase in body weight. As a result of the reduced sympathetic nervous activity obtained in the present study (Table 2) and other reports [7, 12], it appears that the exercise intensity in each patient might be spontaneously increased.

This study had some limitations. First, the number of participants was small. Second, a detailed evaluation of the $\mathrm{O}_{2}$ delivery-utilization mismatch in skeletal muscle should have been included. Third, the duration of intervention, especially MCT treatment, was short. Given that MCT treatment is less costly and more appealing, compared with ghrelin infusion therapy, therapeutic MCT regimens that used different doses and durations should have also been conducted. Fourth, a comparison with MCT alone should have been included in the present study's protocol. Although the dose or duration of MCT treatment was different from the present study, Ashitani et al. [44] reported that 2-week MCT treatment with octanoic acid (2.8 g/day) increased the mean BMI $(16.0 \mathrm{~kg} /$ $\mathrm{m}^{2}$ at pre-treatment vs. $16.3 \mathrm{~kg} / \mathrm{m}^{2}$ after 2 week-MCT treatment) in patients with chronic respiratory disease, but the response was lower than that shown in Table 2 of the present study. Therefore, the difference between the studies might be due to the effect of intravenous ghrelin treatment. Based on the results of the present study, a multicenter, randomized controlled study should be conducted in the future to investigate the effects of adding dietary octanoic acid C8:0 to ghrelin treatment in COPD patients.

\section{Conclusions}

Short-term treatment with MCT alone increased the acyl ghrelin levels but maintained the total ghrelin levels. Combination therapy with MCT and ghrelin improved the symptoms and aerobic capacity of underweight patients with advanced COPD. Given that these improvements were related with increased $\mathrm{O}_{2}$ delivery through cardiocirculatory and muscular crosstalk, which resulted in decreased ventilatory demand, acyl ghrelin with MCT administration may be of therapeutic relevance in cachectic patients including those with COPD.

Acknowledgements The authors would like to thank Mr. E Oda (AC Medical Inc., Tokyo, Japan) for his assistance in statistical analysis. The authors would also like to thank the other members of the MCT and Ghrelin Study Group, as follows: Takeshi Ogura (Osaka Antituberculosis Association), Kazuto Hirata (Department of Respiratory Medicine, Graduate School of Medicine, Osaka City University), Yukio Nagasaka (Respiratory Center, Rakuwakai Otowa Hospital), Saburo Sakoda (Department of Neurology, National Hospital Organization Osaka Toneyama Medical Center), Hirano Kenichi (Department of Cardiovascular Medicine, Graduate School of Medicine, Osaka University), Masahide Mori (Department of Respiratory Medicine, National Hospital Organization Osaka Toneyama Medical Center), Takeshi Kuwahara (Department of Pharmacy, National Cerebral and Cardiovascular Center Research Institute), Toshinori Kokawa (Department of Pharmacy, National Cerebral and Cardiovascular Center Research Institute), Hiromi Hashizume (Department of Pharmacy, National Cerebral and Cardiovascular Center Research Institute), Masayoshi Doi 
(Department of Pharmacy, National Hospital Organization Osaka Toneyama Medical Center), and Misato Tsuji (Department of Pharmacy, National Hospital Organization Osaka Toneyama Medical Center).

Author contributions All authors contributed substantially to this article. MK conceived and designed the study, performed the experiments, analyzed the data, and wrote the manuscript. MM and MR conceived and designed the study, performed the experiments, and analyzed the data. KS, YK, KH, and OY conceived and designed the study and performed the experiments. HSP and SR conceived and designed the study and analyzed the data. TK and $\mathrm{KH}$ reviewed the data and edited the manuscript. KK conceived and designed the study. Each author approved the submission of this manuscript for publication.

Funding Intramural Research Fund (25-3-1) for Cardiovascular Diseases of National Cerebral and Cardiovascular Center, and Grant-inAid for Clinical Research from the National Hospital Organization.

\section{Compliance with ethical standards}

Conflict of interest The authors declare that they have no conflicts of interest.

Ethics approval The study was conducted according to the principles of the Declaration of Helsinki and the Good Clinical Practice guidelines, and it was approved by the ethics committee of NHO Osaka Toneyama Medical Center (approval number, 1424). All patients gave written informed consent.

\section{References}

1. Myers J, Prakash M, Froelicher V, Do D, Partington S, Atwood JE (2002) Exercise capacity and mortality among men referred for exercise testing. N Engl J Med 346:793-801

2. Oga T, Nishimura K, Tsukino M, Sato S, Hajiro T (2003) Analysis of the factors related to mortality in chronic obstructive pulmonary disease: role of exercise capacity and health status. Am J Respir Crit Care Med 167:544-549

3. WHO (2018) The top 10 causes of death. https://www.who. int/news-room/fact-sheets/detail/the-top-10-causes-of-death Accessed 2 Jun 2019

4. Latorre R, Sternini C, De Giorgio R, Greenwood-Van Meerveld B (2016) Enteroendocrine cells: a review of their role in brain-gut communication. Neurogastroenterol Motil 28:620-630

5. Kojima M, Hosoda H, Date Y, Nakazato M, Matsuo H, Kangawa $\mathrm{K}$ (1999) Ghrelin is a growth-hormone-releasing acylated peptide from stomach. Nature 402:656-660

6. Nakazato M, Murakami N, Date Y, Kojima M, Matsuo H, Kangawa K, Matsukura S (2001) A role for ghrelin in the central regulation of feeding. Nature 409:194-198

7. Matsumura K, Tsuchihashi T, Fujii K, Abe I, Iida M (2002) Central ghrelin modulates sympathetic activity in conscious rabbits. Hypertension 40:694-699

8. Nagaya N, Kojima M, Uematsu M, Yamagishi M, Hosoda H, Oya H, Hayashi Y, Kangawa K (2001) Hemodynamic and hormonal effects of human ghrelin in healthy volunteers. Am J Physiol Regul Integr Comp Physiol 280:R1483-R1487

9. Nagaya N, Miyatake K, Uematsu M, Oya H, Shimizu W, Hosoda H, Kojima M, Nakanishi N, Mori H, Kangawa K (2001) Hemodynamic, renal, and hormonal effects of ghrelin infusion in patients with chronic heart failure. J Clin Endocrinol Metab 86:5854-5859
10. Nagaya N, Uematsu M, Kojima M, Ikeda Y, Yoshihara F, Shimizu W, Hosoda H, Hirota Y, Ishida H, Mori H, Kangawa K (2001) Chronic administration of ghrelin improves left ventricular dysfunction and attenuates development of cardiac cachexia in rats with heart failure. Circulation 104:1430-1435

11. Tschop M, Smiley DL, Heiman ML (2000) Ghrelin induces adiposity in rodents. Nature 407:908-913

12. Nagaya N, Itoh T, Murakami S, Oya H, Uematsu M, Miyatake K, Kangawa K (2005) Treatment of cachexia with ghrelin in patients with COPD. Chest 128:1187-1193

13. Miki K, Maekura R, Nagaya N, Kitada S, Miki M, Yoshimura K, Tateishi Y, Motone M, Hiraga T, Mori M, Kangawa K (2013) Effects of ghrelin treatment on exercise capacity in underweight COPD patients: a substudy of a multicenter, randomized, double-blind, placebo-controlled trial of ghrelin treatment. BMC Pulm Med 13:37

14. Miki K, Maekura R, Nagaya N, Nakazato M, Kimura H, Murakami S, Ohnishi S, Hiraga T, Miki M, Kitada S, Yoshimura K, Tateishi Y, Arimura Y, Matsumoto N, Yoshikawa M, Yamahara K, Kangawa K (2012) Ghrelin treatment of cachectic patients with chronic obstructive pulmonary disease: a multicenter, randomized, double-blind, placebo-controlled trial. PLoS One 7:e35708

15. Nishi Y, Hiejima H, Hosoda H, Kaiya H, Mori K, Fukue Y, Yanase T, Nawata H, Kangawa K, Kojima M (2005) Ingested medium-chain fatty acids are directly utilized for the acyl modification of ghrelin. Endocrinology 146:2255-2264

16. Darling JE, Zhao F, Loftus RJ, Patton LM, Gibbs RA, Hougland JL (2015) Structure-activity analysis of human ghrelin O-acyltransferase reveals chemical determinants of ghrelin selectivity and acyl group recognition. Biochemistry 54:1100-1110

17. El Khoury D, El-Rassi R, Azar S, Hwalla N (2010) Postprandial ghrelin and PYY responses of male subjects on low carbohydrate meals to varied balancing proportions of proteins and fats. Eur J Nutr 49:493-500

18. Kawai K, Nakashima M, Kojima M, Yamashita S, Takakura S, Shimizu M, Kubo C, Sudo N (2017) Ghrelin activation and neuropeptide $\mathrm{Y}$ elevation in response to medium chain triglyceride administration in anorexia nervosa patients. Clin Nutr ESPEN 17:100-104

19. Matsumoto N, Miki K, Tsubouchi H, Sakamoto A, Arimura Y, Yanagi S, Iiboshi H, Yoshida M, Souma R, Ishimoto H, Yamamoto Y, Yatera K, Yoshikawa M, Sagara H, Iwanaga T, Mukae H, Maekura R, Kimura H, Nakazato M, Kangawa K (2015) Ghrelin administration for chronic respiratory failure: a randomized dosecomparison trial. Lung 193:239-247

20. ATS (1995) Standardization of spirometry, 1994 update. Am J Respir Crit Care Med 152:1107-1136

21. Miki K, Tsujino K, Edahiro R, Kitada S, Miki M, Yoshimura K, Kagawa H, Oshitani Y, Ohara Y, Hosono Y, Kurebe H, Maekura R (2018) Exercise tolerance and balance of inspiratory-to-expiratory muscle strength in relation to breathing timing in patients with chronic obstructive pulmonary disease. J Breath Res 12:036008

22. Borg GA (1982) Psychophysical bases of perceived exertion. Med Sci Sports Exerc 14:377-381

23. Hajiro T, Nishimura K, Tsukino M, Ikeda A, Koyama H, Izumi T (1998) Comparison of discriminative properties among diseasespecific questionnaires for measuring health-related quality of life in patients with chronic obstructive pulmonary disease. Am J Respir Crit Care Med 157:785-790

24. Jones PW, Quirk FH, Baveystock CM, Littlejohns P (1992) A selfcomplete measure of health status for chronic airflow limitation. The St. George's Respiratory Questionnaire. Am Rev Respir Dis 145:1321-1327

25. Jones PW (2005) St. George's Respiratory Questionnaire: MCID. Copd 2:75-79 
26. Akamizu T, Sakura N, Shigematsu Y, Tajima G, Ohtake A, Hosoda H, Iwakura H, Ariyasu H, Kangawa K (2012) Analysis of plasma ghrelin in patients with medium-chain acyl-CoA dehydrogenase deficiency and glutaric aciduria type II. Eur J Endocrinol 166:235-240

27. Shrestha R, Hirano KI, Suzuki A, Yamaguchi S, Miura Y, Chen YF, Mizuta M, Chiba H, Hui SP (2017) Change in plasma total, esterified and non-esterified capric acid concentrations during a short-term oral administration of synthetic tricaprin in dogs. Anal Sci 33:1297-1303

28. Shrestha R, Hui SP, Imai H, Hashimoto S, Uemura N, Takeda S, Fuda H, Suzuki A, Yamaguchi S, Hirano K, Chiba H (2015) Plasma capric acid concentrations in healthy subjects determined by high-performance liquid chromatography. Ann Clin Biochem 52:588-596

29. Broom DR, Miyashita M, Wasse LK, Pulsford R, King JA, Thackray AE, Stensel DJ (2017) Acute effect of exercise intensity and duration on acylated ghrelin and hunger in men. J Endocrinol 232:411-422

30. Shiiya T, Ueno H, Toshinai K, Kawagoe T, Naito S, Tobina T, Nishida Y, Shindo M, Kangawa K, Tanaka H, Nakazato M (2011) Significant lowering of plasma ghrelin but not des-acyl ghrelin in response to acute exercise in men. Endocr J 58:335-342

31. Cummings DE, Purnell JQ, Frayo RS, Schmidova K, Wisse BE, Weigle DS (2001) A preprandial rise in plasma ghrelin levels suggests a role in meal initiation in humans. Diabetes 50:1714-1719

32. Itoh $\mathrm{T}$, Nagaya $\mathrm{N}$, Yoshikawa M, Fukuoka A, Takenaka H, Shimizu Y, Haruta Y, Oya H, Yamagishi M, Hosoda H, Kangawa K, Kimura H (2004) Elevated plasma ghrelin level in underweight patients with chronic obstructive pulmonary disease. Am J Respir Crit Care Med 170:879-882

33. Lemarie F, Beauchamp E, Legrand P, Rioux V (2016) Revisiting the metabolism and physiological functions of caprylic acid (C8:0) with special focus on ghrelin octanoylation. Biochimie 120:40-48

34. Akamizu T, Takaya K, Irako T, Hosoda H, Teramukai S, Matsuyama A, Tada H, Miura K, Shimizu A, Fukushima M, Yokode M, Tanaka K, Kangawa K (2004) Pharmacokinetics, safety, and endocrine and appetite effects of ghrelin administration in young healthy subjects. Eur J Endocrinol 150:447-455

35. Tong J, Dave N, Mugundu GM, Davis HW, Gaylinn BD, Thorner MO, Tschop MH, D'Alessio D, Desai PB (2013) The pharmacokinetics of acyl, des-acyl, and total ghrelin in healthy human subjects. Eur J Endocrinol 168:821-828
36. Augustin K, Khabbush A, Williams S, Eaton S, Orford M, Cross JH, Heales SJR, Walker MC, Williams RSB (2018) Mechanisms of action for the medium-chain triglyceride ketogenic diet in neurological and metabolic disorders. Lancet Neurol 17:84-93

37. Schmidt M, Pfetzer N, Schwab M, Strauss I, Kammerer U (2011) Effects of a ketogenic diet on the quality of life in 16 patients with advanced cancer: a pilot trial. Nutr Metab (Lond) 8:54

38. Chiappa GR, Borghi-Silva A, Ferreira LF, Carrascosa C, Oliveira CC, Maia J, Gimenes AC, Queiroga F Jr, Berton D, Ferreira EM, Nery LE (1985) Neder JA (2008) Kinetics of muscle deoxygenation are accelerated at the onset of heavy-intensity exercise in patients with COPD: relationship to central cardiovascular dynamics. J Appl Physiol 104:1341-1350

39. Medeiros WM, Fernandes MC, Azevedo DP, de Freitas FF, Amorim BC, Chiavegato LD, Hirai DM, O'Donnell DE, Neder JA (2015) Oxygen delivery-utilization mismatch in contracting locomotor muscle in COPD: peripheral factors. Am J Physiol Regul Integr Comp Physiol 308:R105-R111

40. Rocha A, Arbex FF, Sperandio PA, Mancuso F, Marillier M, Bernard AC, Alencar MCN, O’Donnell DE, Neder JA (2019) Exercise intolerance in comorbid COPD and heart failure: the role of impaired aerobic function. Eur Respir J 53:1802386

41. Miki K, Maekura R, Kitada S, Miki M, Yoshimura K, Yamamoto H, Kawabe T, Kagawa H, Oshitani Y, Satomi A, Nishida K, Sawa N, Inoue K (2017) Pulmonary rehabilitation for COPD improves exercise time rather than exercise tolerance: effects and mechanisms. Int J Chron Obstruct Pulmon Dis 12:1061-1070

42. Okumura H, Nagaya N, Enomoto M, Nakagawa E, Oya H, Kangawa K (2002) Vasodilatory effect of ghrelin, an endogenous peptide from the stomach. J Cardiovasc Pharmacol 39:779-783

43. Temel JS, Abernethy AP, Currow DC, Friend J, Duus EM, Yan Y, Fearon KC (2016) Anamorelin in patients with non-small-cell lung cancer and cachexia (ROMANA 1 and ROMANA 2): results from two randomised, double-blind, phase 3 trials. Lancet Oncol 17:519-531

44. Ashitani J, Matsumoto N, Nakazato M (2009) Effect of octanoic acid-rich formula on plasma ghrelin levels in cachectic patients with chronic respiratory disease. Nutr J 8:25

Publisher's Note Springer Nature remains neutral with regard to jurisdictional claims in published maps and institutional affiliations. 